\title{
A Representation Theorem for Linear Discrete-Space Systems
}

\author{
IRWIN W. SANDBERG * \\ Department of Electrical and Computer Engineering, \\ The University of Texas at Austin, Austin, TX 78712, USA
}

(Received 22 July 1997)

The cornerstone of the theory of discrete-time single-input single-output linear systems is the idea that every such system has an input-output map $H$ that can be represented by a convolution or the familiar generalization of a convolution. This thinking involves an oversight which is corrected in this note by adding an additional term to the representation.

Keywords: Linear systems; Convolutions; Representation theory

\section{INTRODUCTION}

The cornerstone of the theory of discrete-time single-input singleoutput linear systems is the idea that every such system has an input-output map $H$ that can be represented by an expression of the form

$$
(H x)(n)=\sum_{p=-\infty}^{\infty} h(n, p) x(p)
$$

in which $x$ is the input and $h$ is the system function associated with $H$ in a certain familiar way. It is widely known that this, and a corresponding representation for time-invariant systems in which

\footnotetext{
*Fax: 512-471-5532. E-mail: sandberg@uts.cc.utexas.edu.
} 
$h(n, p)$ is replaced with $h(n-p)$, are discussed in many books (see, for example, [1, p. 70], [2, p. 96]). Almost always it is emphasized that these representations hold for all linear input-output maps $H$. On the other hand, in [3, p. 98] attention is directed to material in [4, p. 58] which shows that certain time-invariant $H$ 's in fact do not have convolution representations. ${ }^{1}$ This writer does not claim that these $H$ 's are necessarily of importance in applications, but he does feel that their existence shows that the analytical ideas in the books are flawed. ${ }^{2}$

One of the main purposes of this note is to show that, under some mild conditions concerning the set of inputs and $H$, (1) becomes correct if an additional term is added to the right side. More specifically, we show that

$$
(H x)(n)=\sum_{p=-\infty}^{\infty} h(n, p) x(p)+\lim _{k \rightarrow \infty}\left(H E_{k} x\right)(n)
$$

for each $n$, in which $h$ has the same meaning as in (1), and $E_{k} x$ denotes the function given by $\left(E_{k} x\right)(p)=x(p)$ for $|p|>k$ and $\left(E_{k} x\right)(p)=0$ otherwise. This holds whenever the input set is the set of bounded functions, the outputs are bounded, and $H$ is continuous. In particular, we see that in this important setting, an $H$ has a representation of the form given by (1) if and only if

$$
\lim _{k \rightarrow \infty}\left(H E_{k} x\right)(n)=0
$$

for all $x$ and $n$. Since this is typically a very reasonable condition for a system map $H$ to satisfy, it is clear that the $H$ 's that cannot be represented using just (1) are rather special.

\footnotetext{
${ }^{1}$ The claim in [3] that there exists a time-invariant causal $H$ that has no convolution representation is correct, but it may not be clear that the argument given there actually shows this. Specifically, it may not be clear from what is said in [3] that the pertinent map constructed there is causal and time-invariant. However, it is not difficult to modify what is said so that it establishes the claim (see $[4$, p. 58] or the proof of the related proposition in our appendix).

${ }^{2}$ The oversight in the books is due to the lack of validity of the interchange of the order of performing a certain infinite sum and then applying $(H \cdot)(n)$. The infinite sum at issue clearly converges pointwise, but that is not enough to justify the interchange. (From another viewpoint, the oversight is due to the invalid conclusion that superposition always holds in the case of any linear system with an excitation that can be written as a sum of a countably infinite number of excitations.)
} 
Our results concerning $H$ are given in the following section, in which the setting is more general in that we address $H$ 's for which inputs and outputs depend on an arbitrary finite number of variables. This case is of interest in connection with, for example, image processing. We also consider $H$ 's for which inputs and outputs are defined on just the non-negative integers because that case too arises often in applications. In that setting the situation with regard to the need for an additional term in the representation is different: no additional term is needed for causal maps $H$.

\section{REPRESENTATION RESULT}

\subsection{Preliminaries}

Let $m$ be a positive integer, let $\mathcal{Z}$ be the set of all integers, and let $\mathcal{Z}_{+}$denote the set of non-negative integers. Let $D$ stand for either $\mathcal{Z}^{m}$ or $\mathcal{Z}_{+}^{m}$. Let $F$ be either the set of real numbers or the set of complex numbers, and let $\ell_{\infty}(D)$ denote the normed linear space of bounded $F$-valued functions $x$ defined on $D$, over the field $F$, with the norm $\|\cdot\|$ given by $\|x\|=\sup _{\alpha \in D}|x(\alpha)|$.

For each positive integer $k$, let $c_{k}$ stand for the discrete hypercube $\left\{\alpha \in D:\left|\alpha_{j}\right| \leq k \forall j\right\} \quad\left(\alpha_{j}\right.$ is the $j$ th component of $\alpha$ ), and let $\ell_{1}(D)$ denote the set of $F$-valued maps $g$ on $D$ such that

$$
\sup _{k} \sum_{\beta \in c_{k}}|g(\beta)|<\infty
$$

For each $g \in \ell_{1}(D)$ the sum $\sum_{\beta \in c_{k}} g(\beta)$ converges to a finite limit as $k \rightarrow \infty$, and we denote this limit by $\sum_{\beta \in D} g(\beta)$.

Define maps $Q_{k}$ and $E_{k}$ from $\ell_{\infty}(D)$ into itself by $\left(Q_{k} x\right)(\alpha)=x(\alpha)$, $\alpha \in c_{k}$ and $\left(Q_{k} x\right)(\alpha)=0$ otherwise, and $\left(E_{k} x\right)(\alpha)=x(\alpha), \alpha \notin c_{k}$ and $\left(E_{k} x\right)(\alpha)=0$ otherwise.

In the next section $H$ stands for any linear map from $\ell_{\infty}(D)$ into itself that satisfies the condition that

$$
\sup _{k}\left|\left(H Q_{k} u\right)(\alpha)\right|<\infty
$$


for each $u \in \ell_{\infty}(D)$ and each $\alpha \in D$. This condition is met whenever $H$ is continuous because then $\left|\left(H Q_{k} u\right)(\alpha)\right| \leq\left\|H Q_{k} u\right\| \leq\|H\| \cdot\left\|Q_{k} u\right\| \leq$ $\|H\| \cdot\|u\|^{3}$

\subsection{Our Theorem}

In the following theorem, $h(\cdot, \beta)$ for each $\beta \in D$ is defined by $h(\cdot, \beta)=$ $H \delta_{\beta}$, where $\left(\delta_{\beta}\right)(\alpha)=1$ for $\alpha=\beta$ and $\left(\delta_{\beta}\right)(\alpha)$ is zero otherwise. Of course $h(\cdot, \beta)$ is the response of $H$ to a unit "impulse" occurring at $\alpha=\beta$.

THEOREM For any $H$ as described, and for each $\alpha \in D$ and each $x \in \ell_{\infty}(D)$,

(i) $g$ defined on $D$ by $g(\beta)=h(\alpha, \beta) x(\beta)$ belongs to $\ell_{1}(D)$;

(ii) $\lim _{k \rightarrow \infty}\left(H E_{k} x\right)(\alpha)$ exists and is finite;

(iii) we have

$$
(H x)(\alpha)=\sum_{\beta \in D} h(\alpha, \beta) x(\beta)+\lim _{k \rightarrow \infty}\left(H E_{k} x\right)(\alpha) .
$$

Proof Let $\alpha \in D$ and $x \in \ell_{\infty}(D)$ be given. By the linearity of $H$ and the definition of $Q_{k}$,

$$
\sum_{\beta \in c_{k}} h(\alpha, \beta) u(\beta)=H\left(\sum_{\beta \in c_{k}} u(\beta) \delta_{\beta}\right)(\alpha)=H\left(Q_{k} u\right)(\alpha)
$$

for each $k$ and each $u \in \ell_{\infty}(D)$. In particular, for $u$ given by $u(\beta)=$ $h(\alpha, \beta)^{*} /|h(\alpha, \beta)|$ if $h(\alpha, \beta) \neq 0\left[h(\alpha, \beta)^{*}\right.$ is the complex conjugate of $h(\alpha, \beta)]$ and $u(\beta)=0$ otherwise $\forall \beta$,

$$
\sum_{\beta \in c_{k}}|h(\alpha, \beta)|=H\left(Q_{k} u\right)(\alpha)
$$

for each $k$. Thus, by (2), $h(\alpha, \cdot)$ belongs to $\ell_{1}(D)$ and so does $g$ of (i) of the theorem. By (i) the extreme left and right sides of (3) with

\footnotetext{
${ }^{3}$ Here we have used the well-known fact that boundedness and continuity are equivalent for a linear operator between normed linear spaces.
} 
$u=x$ converge as $k \rightarrow \infty$, and one has

$$
\sum_{\beta \in D} h(\alpha, \beta) x(\beta)=\lim _{k \rightarrow \infty} H\left(Q_{k} x\right)(\alpha) .
$$

Since

$$
H\left(E_{k} x\right)(\alpha)=(H x)(\alpha)-H\left(Q_{k} x\right)(\alpha)
$$

for each $k$, it is clear that (ii) holds. Finally,

$$
\lim _{k \rightarrow \infty} H\left(E_{k} x\right)(\alpha)=(H x)(\alpha)-\lim _{k \rightarrow \infty} H\left(Q_{k} x\right)(\alpha),
$$

which, together with (4), completes the proof.

We note that for $D=\mathcal{Z}_{+}^{m}$ and $H$ causal in the usual sense (see the appendix), the term $\lim _{\mathrm{k} \rightarrow \infty}\left(H E_{k} x\right)(\alpha)$ is always zero. In the appendix an extension result for shift-invariant maps defined on $\ell_{\infty}\left(\mathcal{Z}^{m}\right)$ is given from which it follows that there are maps $H$ for which the additional term is not always zero.

\section{APPENDIX: AN EXTENSION PROPOSITION}

We begin with some additional preliminaries: Let $M$ denote a linear manifold in $\ell_{\infty}\left(\mathcal{Z}^{m}\right)$ that is closed under translation in the sense that $T_{\beta} M=M$ for each $\beta \in \mathcal{Z}^{m}$, where $T_{\beta}$ is the usual shift map defined on $M$ for each $\beta \in \mathcal{Z}^{m}$ by $\left(T_{\beta} x\right)(\alpha)=x(\alpha-\beta), \alpha \in \mathcal{Z}^{m}$. Assume also that $y \in M$ implies that $z \in M$, where $z(\alpha)=y(\alpha)$ for $\alpha_{j} \leq 0 \forall j$, and $z(\alpha)=0$ otherwise. We do not rule out the possibility that $M=\ell_{\infty}\left(\mathcal{Z}^{m}\right)$.

Let $A$ be a linear map of $M$ into $\ell_{\infty}\left(\mathcal{Z}^{m}\right)$. Such an $A$ is shiftinvariant if

$$
(A x)(\alpha-\beta)=\left(A T_{\beta} x\right)(\alpha), \quad \alpha \in \mathcal{Z}^{m}
$$

for each $\beta \in \mathcal{Z}^{m}$ and $x \in M$. The map $A$ is causal if

$$
x(\alpha)=y(\alpha) \text { whenever } \alpha_{j} \leq \beta_{j} \forall j \Longrightarrow(A x)(\beta)=(A y)(\beta)
$$


for each $\beta \in \mathcal{Z}^{m}$ and every $x$ and $y$ in $M$. It is bounded if $\|A\|_{M}:=$ $\sup \{\|A x\|: x \in M,\|x\| \leq 1\}<\infty$, in which $\|\cdot\|$ is the norm in $\ell_{\infty}\left(\mathcal{Z}^{m}\right)$. Our result is the following.

Proposition Let $A$ be shift invariant and bounded. Then there exists $a$ bounded linear shift-invariant map $B$ from $\ell_{\infty}\left(\mathcal{Z}^{m}\right)$ into itself that extends $A$ in the sense that $B$ is causal if $A$ is causal and $B x=A x$, $x \in M$.

Proof By the shift invariance of $A$, we have $(A x)(\alpha)=\left(A T_{-\alpha} x\right)(0)$ for all $\alpha$ and all $x \in M$. The map $(A \cdot)(0)$ is a bounded linear functional on $M$, because

$$
\begin{aligned}
|(A y)(0)| & =\left|\left(A T_{-\alpha} T_{\alpha} y\right)(0)\right|=\left|\left(A T_{\alpha} y\right)(\alpha)\right| \\
& \leq \sup _{\beta}\left|\left(A T_{\alpha} y\right)(\beta)\right| \leq\|A\|_{M} \cdot\left\|T_{\alpha} y\right\|=\|A\|_{M} \cdot\|y\|
\end{aligned}
$$

for $y \in M$. When $A$ is causal, $(A \cdot)(0)$ has the property that $(A y)(0)=0$ for any $y \in M$ for which $y(\alpha)=0$ for $\alpha_{j} \leq 0 \forall j$. By the Hahn-Banach theorem [5, pp. 178 and 181] there is a bounded linear functional $\mathcal{F}$ that extends $(A \cdot)(0)$ to all of $\ell_{\infty}\left(\mathcal{Z}^{m}\right)$. Set $\mathcal{G}=\mathcal{F}$ if $A$ is not causal, and if $A$ is causal define $\mathcal{G}$ on $\ell_{\infty}\left(\mathcal{Z}^{m}\right)$ by $\mathcal{G} y=\mathcal{F} P y$ where $P$ is the linear operator given by $(P y)(\alpha)=y(\alpha), \alpha_{j} \leq 0 \forall j$ and $(P y)(\alpha)=0$ for $\alpha_{j}>0$ for some $j$. Define $B$ on $\ell_{\infty}\left(\mathcal{Z}^{m}\right)$ by $(B x)(\alpha)=\mathcal{G} T_{-\alpha} x$. It is easy to check that $B$ is a linear shift-invariant bounded map into $\ell_{\infty}\left(\mathcal{Z}^{m}\right)$, that $B$ extends $A$ to $\ell_{\infty}\left(\mathcal{Z}^{m}\right)$, and that $B$ is causal if $A$ is causal. ${ }^{4}$ This completes the proof.

Since the set $L$ of elements $x$ of $\ell_{\infty}\left(\mathcal{Z}^{m}\right)$ such that $x(\alpha)$ approaches a limit as $\max _{j}\left\{\alpha_{j}\right\} \rightarrow-\infty$ is a linear manifold that is closed under translation, and since

$$
(A x)(\alpha)=\lim _{\max _{j}\left\{\beta_{j}\right\} \rightarrow-\infty} x(\beta)
$$

defines a shift-invariant bounded causal linear map of $L$ into $\ell_{\infty}\left(\mathcal{Z}^{m}\right)$, it follows from our proposition that there exist maps $H$, even causal time-invariant maps $H$, of the kind addressed by our theorem for

\footnotetext{
${ }^{4}$ It is also true that $B$ can be chosen so that it preserves the norm of $A$, in the sense that $\|B\|=\|A\|_{M}$.
} 
which the term $\lim _{k \rightarrow \infty}\left(H E_{k} x\right)(\alpha)$ is not always zero. More explicitly, the associate $B$ via our proposition of the $A$ just described satisfies $\lim _{k \rightarrow \infty}\left(B E_{k} x\right)(\alpha)=\lim _{\max _{j}\left\{\beta_{j}\right\} \rightarrow-\infty} x(\beta)$ for $x \in L$. And an example of an $H$ of the type addressed by the theorem for which the additional term is not always zero and $H$ is not shift invariant is obtained by adding to this $B$ any linear bounded map of $\ell_{\infty}\left(\mathcal{Z}^{m}\right)$ into itself that is not shift invariant and has a representation without an additional term.

A proposition similar to the one above can be given to show that there are bounded linear continuous-time time-invariant inputoutput maps that do not possess certain convolution representations.

\section{References}

[1] J.G. Proakis and D.G. Manolakis, Digital Signal Processing, second edition, Macmillan, New York, 1992.

[2] L.B. Jackson, Signals, Systems, and Transforms, Addison-Wesley, New York, 1991.

[3] S.P. Boyd, Volterra Series: Engineering Fundamentals, Dissertation, University of California, Berkeley, 1985.

[4] L.V. Kantorovich and G.P. Akilov, Functional Analysis, Pergamon, Oxford, 1982.

[5] G. Bachman and L. Narici, Functional Analysis, Academic Press, New York, 1966. 


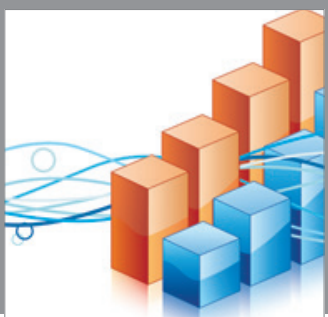

Advances in

Operations Research

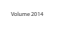

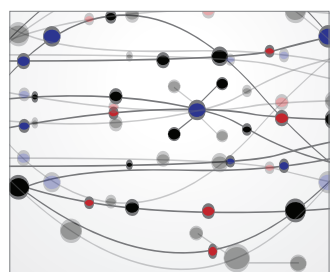

\section{The Scientific} World Journal
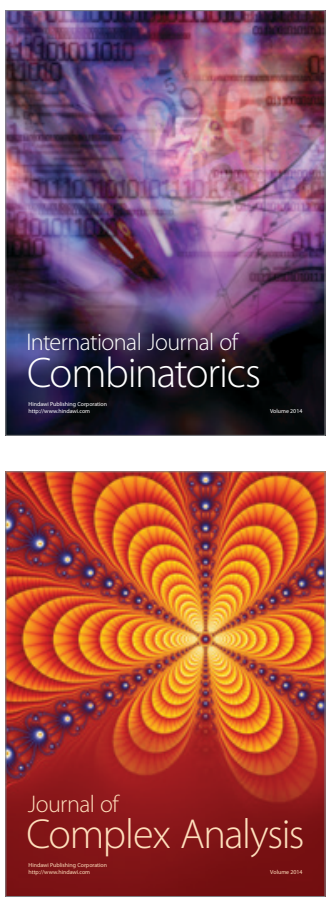

International Journal of

Mathematics and

Mathematical

Sciences
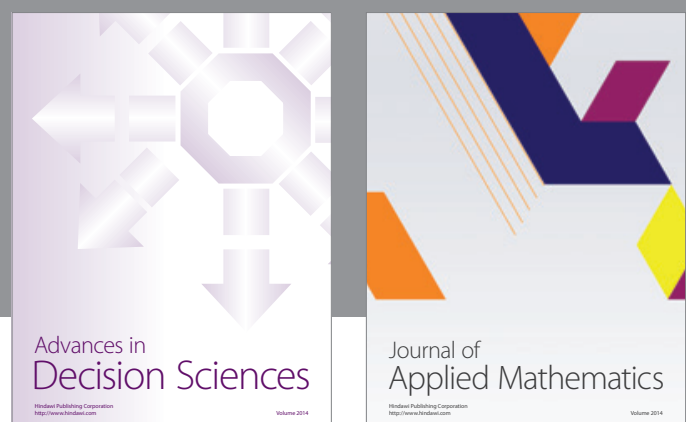

Journal of

Applied Mathematics
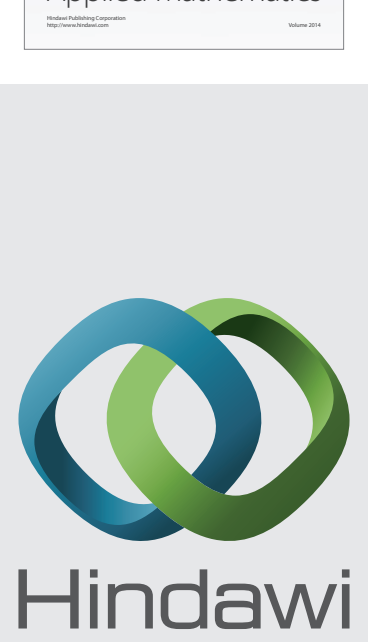

Submit your manuscripts at http://www.hindawi.com
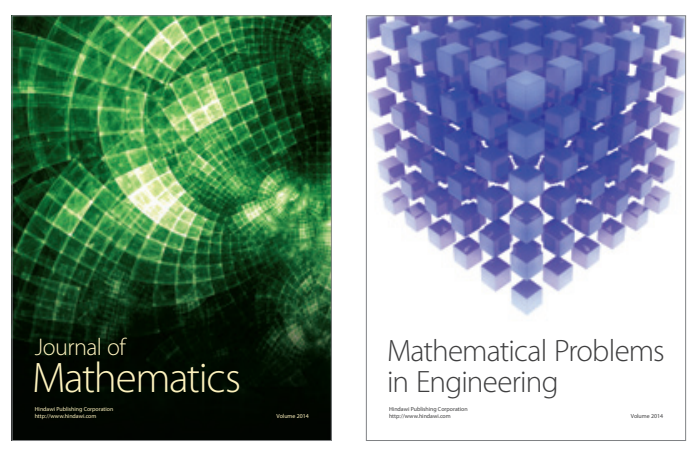

Mathematical Problems in Engineering
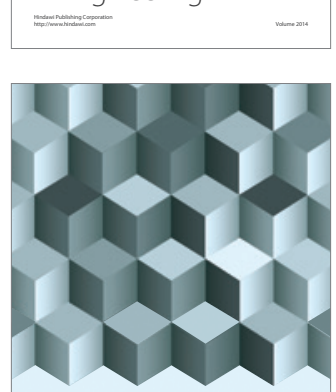

Journal of

Function Spaces
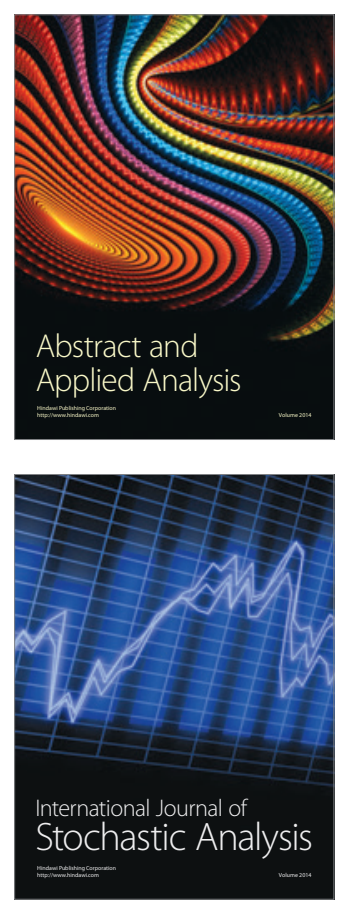

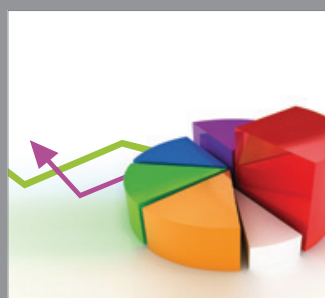

ournal of

Probability and Statistics

Promensencen
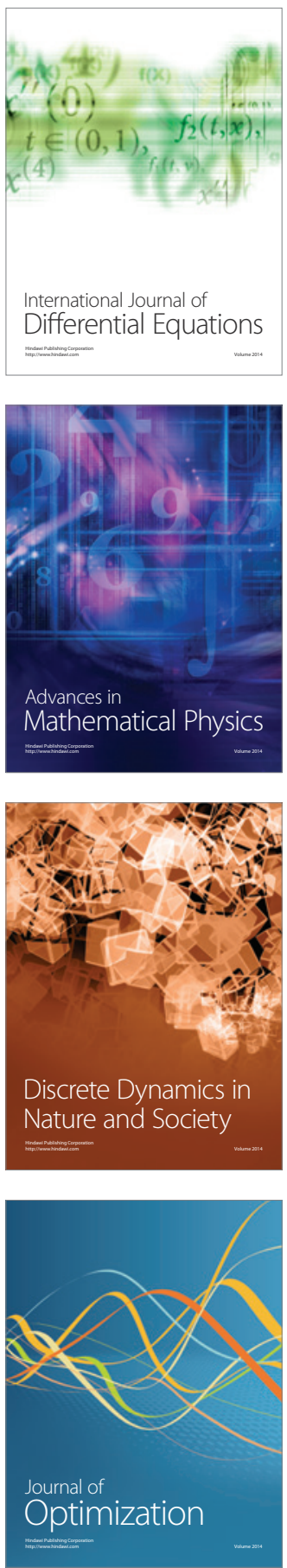